\title{
Acciones sobre los determinantes sociales de la salud en Venezuela
}

\section{Actions on social determinants of health in Venezuela}

\section{Tulia Hernández Muñoz}

Máster en Evaluación de Impacto Ambiental. Profesora. Instituto de Altos Estudios "Dr. Arnoldo Gabaldon". Maracay, Venezuela.

\section{RESUMEN}

Se revisa desde la perspectiva de la ciudadanía, del sistema de salud y de la acción sectorial e intersectorial, los avances y desafíos en las intervenciones sobre los determinantes sociales de la salud en Venezuela, analizados bajo el modelo descrito por la Comisión de Determinantes Sociales de la Salud de la OMS, para valorar las desigualdades en salud, atendiendo y cumpliendo las recomendaciones de la citada comisión. Se presentan ejemplos de las acciones sanitarias y extra sanitarias que se han venido desarrollando en el país en pro de impulsar políticas públicas para disminuir la exposición y vulnerabilidad diferencial a los determinantes sociales de la salud con la intención de reducir las inequidades. Se relacionan estas acciones con modalidad de política de salud asumida en base a principios de equidad y justicia social. Se identifican desafíos en áreas específicas como los accidentes de tránsito y hechos violentos, el cuidado del medio ambiente y de la salud de los trabajadores. No obstante, para cerrar brechas sanitarias y lograr mejores y más rápidos resultados, es necesario fortalecer las capacidades técnicas del talento humano, realizar mayores esfuerzos a nivel extra sanitario para disminuir el burocratismo y la corrupción; y lograr, a nivel sanitario, la asignación de recursos y definición de programas de acuerdo a las necesidades.

Palabras clave: Determinantes sociales de la salud, políticas de intervención, Venezuela.

\section{ABSTRACT}


From the perspective of the population, the health system and the sectoral and intersectoral actions, a review was made of the advances and challenges found in the interventions on the social determinants of health in Venezuela, which were analyzed according to the WHO Commission on Social Determinants of Health model to evaluate health inequalities based on the recommendations of this commission. Examples of health and non-health actions that are being taken in the country were given. These actions were aimed at encouraging public policies to decrease differential exposure and vulnerability to social determinants of health, for the purpose of reducing inequities. These actions were listed by health policy modality based on equity and social justice principles. The challenges in specific areas such as traffic accidents and violent events, environmental protection and occupational health were shown. Nevertheless, closing gaps in health and achieving better and expedite results demand to strengthen the technical capabilities of human resources, to make greater efforts to reduce corruption and red tape in the health-unrelated sphere, and to accomplish, at healthcare level, the allocation of necessary resources and the definition of need-linked programs.

Key words: Social determinants of health, intervention policies, Venezuela.

\section{INTRODUCCIÓN}

Infantes, adolescentes, mujeres, personas con discapacidad, población indígena, población rural y población en situación de extrema pobreza son reconocidos como grupos tradicionalmente excluidos, por haber tenido menos acceso a los recursos sanitarios básicos y al sistema de salud en general.

Ante ello, el gobierno venezolano ha respondido mediante la aplicación progresiva de políticas públicas orientadas al disfrute pleno y efectivo de derechos sociales, fundamentales en la búsqueda de la justicia social y con ello la disminución de brechas. Se ha ido más allá de los servicios de atención y de las enfermedades, hacia la promoción de la calidad de vida mediante el desarrollo de intervenciones orientadas a la transformación de los determinantes económicos, sociales, culturales y geográficos que influyen en la situación de salud. Esto se plantea por primera vez en el país desde el eje de Equilibrio Social contemplado en el Plan de Desarrollo Social y Económico de la Nación, 2001-2007 (MPD, 2001) y se ratifica en el Proyecto Nacional Simón Bolívar: Primer Plan Socialista de la Nación 2007$2013^{2}$ (GBV, 2010). Este trabajo aborda desde la perspectiva de la ciudadanía, del sistema de salud y la acción intersectorial, las acciones sobre los determinantes sociales de la salud (DSS), sus avances y desafíos en Venezuela, analizadas bajo el modelo conceptual descrito por la Comisión de Determinantes Sociales de la Salud (CDSS) de la OMS, para valorar las desigualdades en salud y atendiendo a las recomendaciones planteadas en el informe final de la CDSS.

\section{CONTEXTO Y ACCIONES SOBRE LOS DETERMINANTES SOCIALES DE LA SALUD}

Con base en los artículos 5, 6, 83, 84 y 184 de la Constitución de la República Bolivariana de Venezuela (CRBV) ${ }^{3}$, y leyes como la Ley Orgánica del Poder Ciudadano (LOPC) ${ }^{4}$, Ley de los Consejos Locales de Planificación Pública (LCLPP) ${ }^{5}$, 
Ley Orgánica del Poder Público Municipal (LOPPM) ${ }^{6}$ y Ley Orgánica de los Consejos Comunales (LOCC), ${ }^{7}$ que promueven la participación en la gestión pública, se han generando espacios para que la comunidad participe activamente. En un grado cada vez mayor, la comunidad tiene la facultad de participar de manera activa y protagónica en las decisiones que afectan sus condiciones de vida, a través de los Consejos Comunales (CC), que por ley se definen como: "... instancias de participación, articulación e integración entre las diversas organizaciones comunitarias, grupos sociales y los ciudadanos y ciudadanas, que permiten al pueblo organizado ejercer directamente la gestión de las políticas públicas y proyectos orientados a responder a las necesidades y aspiraciones de las comunidades en la construcción de una sociedad de equidad y justicia social" 6 (LOCC, 2009). Actualmente sobrepasan de diez mil, el número de CC legalmente constituidos, los cuales se benefician de asignaciones presupuestarias a proyectos para atender problemas de las comunidades, como viviendas, abastecimiento de agua potable, escuelas, canchas deportivas, vialidad, producción y distribución de alimentos, definidos y priorizados por ellos.

Por su parte, el sector de la salud a pesar de todos los logros alcanzados sigue mostrando debilidades, no solo para atender si no para entender los problemas sociales que impactan la salud de la población. Se aprecia deficiencia de recurso humano capacitado, prevalencia de modelo medicalizado, fragmentación del sector, dificultades para el trabajo intersectorial articulado y presupuesto destinado mayoritariamente al gasto en recursos humanos y funcionamiento de hospitales. Además, baja asignación presupuestaria para las acciones de promoción de estilos y condiciones de vida saludables y de atención al medio ambiente. No obstante, el sector tiene entre sus fortalezas una férrea voluntad política por transformarlo en un sistema basado en la atención primaria de salud, con énfasis en la promoción y prevención, conformado en redes de salud imbricadas en redes sociales, con alta participación ciudadana corresponsable, puntos claves para la acción intersectorial.

Las intervenciones que se han venido desarrollando en Venezuela en pro de impulsar políticas públicas saludables, han sido denominadas Misiones Sociales, ${ }^{8}$ a las cuales se destinan recursos financieros ordinarios y recursos financieros producto de los excedentes en el precio del petróleo. Las misiones están dirigidas a disminuir la exposición y vulnerabilidad diferencial a los DSS, a través de la ejecución de acciones sanitarias y extra sanitarias que toman en cuenta las causas más profundas de las inequidades en salud.

Con la finalidad de presentar algunas de las acciones enmarcadas dentro de la política nacional para disminuir desigualdades e iniquidades, estas se organizan en el presente trabajo en cuatro grupos: 1 . Acciones extra sanitarias, en la búsqueda de la reducción de los determinantes estructurales. 2 . Acciones extra sanitarias sobre determinantes intermedios. 3. Acciones extra sanitaria para revertir los efectos de la posición socioeconómica y 4 . Acciones sanitarias con efecto sobre los determinantes intermedios.

\section{Acciones extra sanitarias, en la búsqueda de la reducción de los determinantes estructurales}

Se encuentran las Misiones Educativas como la Misión Robinson I, que nace en 2003, programa masivo de alfabetización, para más de un millón de venezolanos distribuidos por todo el territorio nacional, en aras de procurar su participación, por lo que se logró la declaración por parte de la UNESCO como territorio libre de analfabetismo. A esta misión le siguió la Misión Robinson II, para alcanzar el grado de educación primaria de estos compatriotas. En el mismo año, surge la Misión Ribas, programa educativo para garantizar la continuidad de estudios de quienes no 
han logrado ingresar o culminar sus estudios desde la tercera etapa del nivel de educación básica, hasta el nivel de educación media diversificada y profesional. Y finalmente, la Misión Sucre, la cual tiene por objetivo potenciar la sinergia institucional y la participación comunitaria, para garantizar el acceso a la educación universitaria a todos los bachilleres sin cupo en universidades tradicionales y transformar su condición de excluidos del subsistema de educación superior. ${ }^{8}$

En este grupo, también se encuentran las misiones para el trabajo socioproductivo, ejemplos de ellas son: Misión Ché Guevara, que surge en 2007. El objetivo de este programa es la formación, capacitación y organización laboral sustentables, dentro del objetivo supremo de alcanzar la Misión Cristo: Pobreza y Miseria Cero en el 2021 basada en el alcance de las Metas del Milenio trazadas mediante acuerdo en el seno de la Organización de las Naciones Unidas (ONU) y en la elevación de la calidad de vida en general de toda la población venezolana. La Misión Piar, nace en el 2003, su objetivo es incluir al sector de la pequeña minería en el Plan de Desarrollo Económico y Social de la Nación, (en los ejes económico, social, político, territorial e internacional), promoviendo la organización y participación ciudadana del pequeño minero. ${ }^{8}$

En estas acciones se asume a la educación y al trabajo como procesos fundamentales para la justicia social, se reconoce el carácter estratégico de la educación para el desarrollo humano integral sustentable, la soberanía nacional y la construcción de una sociedad cohesionada, democrática y participativa, por ello se ha masificado la educación en todos sus niveles, a través de la desconcentración del sistema y la creación de nuevas modalidades, alternativas y oportunidades de estudio y trabajo en todo el territorio nacional.

\section{Acciones extra sanitarias sobre algunos de los determinantes intermedios}

Se puede presentar como ejemplos a la Misión Ciencia, la cual pretende el uso extensivo e intensivo del conocimiento en función del desarrollo endógeno, promoviendo el desarrollo científico y tecnológico a través de la interacción de todos los sectores productivos del país; y la Misión Árbol, que tiene el fin favorecer el equilibrio ecológico y la recuperación de espacios degradados en todo el territorio nacional, mediante reforestaciones con fines protectores, agroforestales y comerciales-industriales, como estrategia de manejo integral y uso sustentable de las cuencas hidrográficas. ${ }^{8}$

Por otra parte, están la Misión Villanueva que surge en el 2007 y tiene como principal objetivo la redistribución de la población sobre el espacio, para que las familias vivan mejor. Su finalidad es sustituir ranchos y casas en condiciones precarias por modernas edificaciones, con todas las facilidades de servicios y cambiar la imagen urbana en las principales ciudades del país, y enfrentar así la elevada demanda de inmuebles; y la Misión Zamora, cuyo origen en 2001 es concebido para reorganizar la tenencia y uso de las tierras ociosas con vocación agrícola para erradicar el latifundio, promover el desarrollo del medio rural en los ejes estratégicos del país y garantizar la seguridad agroalimentaria de la población a través del desarrollo de una agricultura sustentable. Persigue la transformación de las tierras en unidades económicas productivas, e integra todo este proceso en las políticas agroalimentarias establecidas, para lo cual se cuenta con la Misión Alimentación, iniciada en 2004 con el objetivo principal de comercializar y mercadear productos alimentarios de primera necesidad. Persigue favorecer la seguridad alimentaria de la población venezolana de escasos recursos económicos. ${ }^{8}$

http://scielo.sld.cu 
Y finalmente en este grupo, la Misión Revolución Energética, puesta en marcha a finales del 2006, para una distribución más justa del potencial energético, con una visión ambientalista que propone alcanzar la eficiencia energética a través del ahorro del consumo de energía y el aprovechamiento de fuentes alternativas, como la eólica y la solar. Toma en cuenta la importancia de estas fuentes para garantizar necesidades fundamentales de alimentación, salud y educación. ${ }^{8}$

\section{Acciones extra sanitarias para revertir los efectos de la posición socioeconómica}

Entre estas se puede mencionar la Misión Madres del Barrio, la cual tiene por objetivo apoyar a las amas de casa que se encuentren en estado de necesidad, a fin de que logren, junto con sus familias, mejorar la situación de pobreza extrema y prepararse para salir de la pobreza en su comunidad, mediante la incorporación de programas sociales y misiones, el acompañamiento comunitario y el otorgamiento de una asignación económica. Por otra parte se cuenta con la Misión Negra Hipólita, iniciada en el 2006, para rescatar, reivindicar y garantizar los derechos de las personas en situación de calle y de la población que vive en pobreza extrema. Está dirigida a combatir la marginalidad. El funcionamiento de esta misión es garantizado por los Comités de Protección Social, organizaciones comunitarias que diagnostican la situación social en su ámbito territorial. La Misión Niños y Niñas del Barrio, surge a partir del 2008, con el objetivo de atender las necesidades y defender a los niños, niñas y adolescentes en situación de calle. ${ }^{8}$

En cuanto a vivienda, en el 2004 nace la Misión Hábitat, destinada a crear soluciones habitacionales de manera inmediata, de forma articulada con los gobiernos locales y las instituciones que trabajan en el sector. No solo abarca la construcción de la vivienda sino sobre todo en el desarrollo del hábitat integral, donde se disponga de todos los servicios. ${ }^{8}$

Adicionalmente, en este grupo está la Misión Guaicaipuro nacida en 2003, su objetivo general es restituir los derechos de los Pueblos Indígenas de acuerdo a la CRBV. Surge en el marco del reconocimiento a la existencia de los pueblos y comunidades indígenas, su organización social, política y económica, sus culturas, usos y costumbres, idiomas y religiones, así como hábitat y derechos originarios sobre las tierras que ancestral y tradicionalmente ocupan y que son necesarias para desarrollar y garantizar sus formas de vida. ${ }^{8}$

\section{Acciones sanitarias con efecto sobre los determinantes intermedios}

Para asegurar el cuidado preventivo y curativo de la población hace siete años se creó la Misión Barrio Adentro I a fin de consolidar la atención primaria como prioridad en la política de salud, para dar respuestas a las necesidades sociales de la población. Tiene como principios la equidad, universalidad, accesibilidad, gratuidad, transectorialidad, pertinencia cultural, participación, justicia y corresponsabilidad social. El objetivo general es garantizar el acceso a los servicios de salud de la población excluida, mediante un modelo de gestión de salud integral orientado al logro de una mejor calidad de vida, mediante la creación de Consultorios y Clínicas Populares, además de los Hospitales del Pueblo, dentro de las comunidades de poco acceso a los ya existentes. En el 2005, se crea la Misión Barrio Adentro II, constituye el segundo nivel de atención para brindar servicio integral gratuito a todos los ciudadanos a través de Centros de Alta Tecnología (CAT), Centros de Diagnóstico Integral (CDI) y las Salas de Rehabilitación Integral (SRI). 
Más adelante, se implementa la Misión Barrio Adentro III que consiste en la modernización de la red hospitalaria del país y, a diferencia de las Misiones Barrio Adentro I y II, utiliza la red tradicional de hospitales para abrir esta tercera fase como un elemento fundamental en la composición del Sistema Público Nacional de Salud. Se enfoca hacia dos componentes: primero, la modernización tecnológica de equipos médicos y un segundo componente dirigido a la remodelación, ampliación y mejora de la infraestructura hospitalaria (GBV, 2010). Y finalmente, Barrio adentro IV, una red de Hospitales Especializados, actualmente la mayoría en fase de construcción, a excepción del Hospital Cardiológico Infantil Latinoamericano "Dr. Gilberto Rodríguez Ochoa", inaugurado en 2006, atiende a población de 0 a 18 años con malformaciones cardíacas congénitas. ${ }^{8}$

En 2004, nace la Misión Milagro con motivo de la expectativa generada en la población venezolana, a raíz de la ampliación de las coberturas de servicios de salud a través de la Misión Barrio Adentro, mediante la cual se comenzó a detectar la inmensa deuda social existente en la población excluida en aspectos relacionados a trastornos visuales degenerativos, limitantes de su autonomía, sobre todo en la población de la tercera edad. Con esta misión se ha logrado incluir a un conjunto de personas que históricamente fueron excluidas de la atención y apartadas de la vida social y productiva por padecer de un problema solucionable a bajo costo, de manera rápida y efectiva, y se ha logrado la independencia de los pacientes y de su núcleo familiar. ${ }^{8}$

La Misión Sonrisa, surge a partir del año 2006, siendo la primera vez que se asume como política de Estado otorgar prótesis dentales a la población para rescatar las sonrisas y restituir la autoestima de millones de venezolanos. Posteriormente, en el 2008 surge la Misión José Gregorio Hernández, con el objetivo de llevar atención primaria a todas las personas que padezcan algún tipo de discapacidad, acompañado de un estudio para determinar las causas, aplicación programas preventivos en casos hereditarios/genéticos y puesta en marcha estrategias de atención, tanto para el afectado como para su familia y entorno social. Finalmente, se activa a finales de 2009, la Misión Niño Jesús, que tiene como objetivo principal desarrollar planes, proyectos y programas que permitan mejorar la calidad de vida y salud de la población materno-infantil. ${ }^{8}$

La tabla relaciona la modalidad de la política de salud asumida con las acciones desarrolladas, basadas en los principios de equidad y justicia social, bajo una mirada a los DSS. 
Tabla. Políticas e intervenciones sobre los determinantes sociales de la salud en

Venezuela

\begin{tabular}{|c|c|c|c|}
\hline \multirow{2}{*}{$\begin{array}{l}\text { Modalidad } \\
\text { política }\end{array}$} & \multicolumn{3}{|c|}{ Intervenciones } \\
\hline & $\begin{array}{l}\text { Para reducir } \\
\text { los } \\
\text { determinantes } \\
\text { estructurales }\end{array}$ & $\begin{array}{l}\text { Sobre los } \\
\text { determinantes } \\
\text { intermedios }\end{array}$ & $\begin{array}{l}\text { Para revertir efectos de la } \\
\text { posición socioeconómica }\end{array}$ \\
\hline $\begin{array}{l}\text { Programas } \\
\text { focalizados }\end{array}$ & & $\begin{array}{l}\text { Misión José } \\
\text { Gregorio } \\
\text { Hernández. }\end{array}$ & $\begin{array}{l}\text { Misión Madres del Barrio. } \\
\text { Misión Negra Hipólita. } \\
\text { Misión Guaicaipuro. } \\
\text { Misión Niñas y Niños del Barrio. }\end{array}$ \\
\hline $\begin{array}{l}\text { Reducción de } \\
\text { brechas }\end{array}$ & $\begin{array}{l}\text { Misión } \\
\text { Educativa. } \\
\text { Misión Ché } \\
\text { Guevara. } \\
\text { Misión Piar. }\end{array}$ & $\begin{array}{l}\text { Misión Barrio } \\
\text { Adentro. } \\
\text { Misión Sonrisa. } \\
\text { Misión Milagro. } \\
\text { Misión Niño } \\
\text { Jesús. } \\
\text { Misión } \\
\text { Alimentación. }\end{array}$ & $\begin{array}{l}\text { Misión Alimentación. } \\
\text { Misión Hábitat. }\end{array}$ \\
\hline Gradientes & Misión Ciencia. & $\begin{array}{l}\text { Misión Villanueva. } \\
\text { Misión Zamora. } \\
\text { Misión Árbol. } \\
\text { Misión Revolución } \\
\text { Energética. }\end{array}$ & \\
\hline
\end{tabular}

\section{DESAFÍOS DESDE LA PERSPECTIVA DEL INFORME FINAL DE LA COMISIÓN DE DETERMINANTES SOCIALES DE LA SALUD DE LA OMS Y SUS RECOMENDACIONES}

La primera recomendación de la Comisión es mejorar las condiciones de vida, ${ }^{9}$ para lo cual se aborda: el bienestar de las niñas y las mujeres, las condiciones en que nacen los niños y el desarrollo de la primera infancia a través de las Misiones Madres del Barrio, Niñas y Niños del Barrio, Niño Jesús, entre otras; la educación de los niños y las niñas mediante las Misiones Educativas (Pre-escolares Simoncitos, Escuelas y Liceos Bolivarianos). Para mejorar las condiciones de vida y de trabajo y brindar protección social, se ha trabajado a través de las misiones Habitat y Ché Guevara, por citar dos ejemplos. Una potente condición en esta línea de acción consiste en la participación ciudadana protagónica. Sin embargo es necesario mejorar la articulación en el trabajo intersectorial, el cual no siempre se corresponde con el nivel esperado.

Algunas de las intervenciones por desarrollar deben ir dirigidas hacia la disminución de los accidentes de tránsito y hechos violentos, el cuidado del medio ambiente y de la salud de los trabajadores, sin olvidar los trabajadores de la salud y el enfoque de género e intercultural en todos los ámbitos.

Se han realizado esfuerzos en la mejoría del desarrollo urbano, la disponibilidad de vivienda digna y servicios básicos, el acceso a los alimentos de los más necesitados y la ordenación adecuada del medio, también se ha creado un sistema de salud basado en la atención primaria y se ha promovido el empleo digno, no obstante, existen debilidades que fueron mencionadas anteriormente. Se requiere implementar acciones para un mayor control sobre establecimientos que promuevan hábitos no saludables, por ejemplo expendios de bebidas alcohólicas o 
de comida sin condiciones higiénicas y establecer políticas que aborden firmemente el cambio climático y la protección de los trabajadores.

Para dar impulso a la segunda recomendación de la Comisión, luchar contra la distribución desigual del poder, dinero y los recursos, ${ }^{9}$ existe un proyecto de país, el Plan Nacional Simón Bolívar, que se va consolidando progresivamente y que sin duda contribuye a lograr la equidad en todas las instancias de la sociedad. La solidez de un sector público, comprometido, capaz y dotado de suficiente financiación, como dice la Comisión, quizás solo es percibida en el sector petrolero, en el cual recae actualmente gran parte de la gestión de programas sociales, esto por debilidad y carencia de rectoría del resto de los sectores. De allí, que se considere necesario fortalecer todas las instancias gubernamentales para que asuman su responsabilidad por la salud, la equidad y el financiamiento de programas para actuar sobre los DSS, así como también fortalecer los sistemas políticos y jurídicos para proteger los derechos humanos y garantizar la justicia social. Respecto a este tema, la sociedad civil cuenta con un marco legal que le favorece en sus derechos humanos y constitucionales, que además establece instancias y medios para que los ciudadanos participen de manera protagónica. No obstante, se requiere mayor trabajo en la conceptualización ética-moral de la equidad, crear conciencias sólidas, comprometidas y responsables a favor de una sociedad más justa.

En cuanto a medir la magnitud del problema, analizarlo y evaluar los efectos, tercera recomendación de la Comisión, ${ }^{9}$ en Venezuela se ha conseguido identificar los problemas con un grado importante de estratificación de la información, aunque no siempre se puede evaluar la magnitud de las inequidades sanitarias a nivel nacional, estatal, municipal, parroquial y de localidad, lo cual es esencial para priorizar y direccionar las acciones. No existen sistemas sólidos de vigilancia de la equidad sanitaria, por lo tanto, aún no se puede evaluar sistemáticamente los efectos de las políticas e intervenciones que se vienen ejecutando. Por lo antes indicado, se hace imprescindible seguir la recomendación de la Comisión en cuanto a invertir en la formación de los responsables de formular las políticas y en los profesionales de la salud, así como en la capacitación de los voceros y voceras de las comunidades en lo que son los DSS. Y por supuesto incluir a los DSS en las líneas prioritarias de investigación en salud pública. Existen las capacidades instaladas de infraestructura física y tecnológica y de facilitadores para desarrollar programas de formación dirigido a profesionales y a comunidades. Es imperativo que las autoridades nacionales apoyen y promuevan esta iniciativa y reconozcan su interés para el desarrollo de políticas públicas saludables, así como también es importante no dejar de considerar una mayor estabilidad laboral de los profesionales y técnicos capacitados para cumplir con esta recomendación, a fin de que los productos de los procesos de formación no se pierdan o sub utilicen.

Mejores y más rápidos resultados para cerrar brechas sanitarias en base a metodologías e intervenciones de eficiencia comprobadas pueden comenzar a desarrollarse; no obstante es necesario: 1 . Fortalecer las capacidades técnicas para el análisis de la situación de salud y la generación de alternativas de solución que se materialicen en políticas, planes y programas destinados a mejorar el estado de salud y calidad de vida de la población; 2 . Realizar mayores esfuerzos a nivel extra sanitario para disminuir el burocratismo y la corrupción y 3 . Lograr, a nivel sanitario, la asignación de recursos y definición de programas de acuerdo a las necesidades para la consecución de políticas moralmente correctas y pragmáticamente efectivas. 


\section{REFERENCIAS BIBLIOGRÁFICAS}

1. Ministerio de Planificación y Desarrollo. Líneas Generales del Plan de Desarrollo Económico y Social de la Nación 2001-2007. Caracas, Venezuela: Ministerio; 2001.

2. Ministerio del Poder Popular de Planificación y Finanzas. Proyecto Nacional Simón Bolívar: Primer Plan Socialista de la Nación 2007-2013. Caracas, Venezuela: Ministerio; 207.

3. Asamblea Nacional Constituyente. Constitución de la República Bolivariana de Venezuela. Gaceta Oficial No 5.453 Extraordinario del 24 de marzo 2000.

4. Asamblea Nacional de la República Bolivariana de Venezuela. Ley Orgánica del Poder Ciudadano (LOPC). Gaceta Oficial № 37.310 del 25 de octubre de 2001.

5. Asamblea Nacional de la República Bolivariana de Venezuela. Ley de los Consejos Locales de Planificación Pública (LCLPP), Gaceta Oficial Nº 37.463 del 12 de junio de 2002.

6. Asamblea Nacional de la República Bolivariana de Venezuela. Ley Orgánica del Poder Público Municipal (LOPPM), Gaceta Oficial Nº 38.204 del 08 de junio de 2005.

7. Asamblea Nacional de la República Bolivariana de Venezuela. Ley Orgánica de los Consejos Comunales. Gaceta Oficial N³8.335 del 28 de diciembre de 2009.

8. Gobierno Bolivariano de Venezuela. Gobierno en línea. Misiones. [sitio en Internet]. [citado $30 \mathrm{Abr}$ 2010t]. Disponible en:

http://www.gobiernoenlinea.ve/miscelaneas/misiones.html

9. Commission on Social Determinants of Health. Closing the gap in a generation: health equity through action on the social determinants of health. Final Report of the Commission on Social Determinants of Health. Geneva: WHO; 2008.

Recibido: 18 de junio de 2010.

Aprobado: 8 de julio de 2010.

Tulia Hernández Muñoz. Instituto de Altos Estudios "Dr. Arnoldo Gabaldon". Maracay, Venezuela.

Telf.: 58-243-2320833. E-mail: tuliah@yahoo.com 